\title{
Verwaltungskommunikation: Perspektive der Politik- und Kommunikationsberatung
}

\section{Klaus Kocks}

„Behördenversagen“ - welch' ein Vorwurf. Ein vernichtendes Urteil. Und alles nur wegen einer Kommunikationspanne eher banaler Natur? Am 07.01.2019 ruft der Chefkommentator der Süddeutschen Zeitung in seinem Leitartikel seinen Lesern ein „Erwachet!“ (Prantl 2019) entgegen. Die Republik soll in Alarmzustand versetzt werden. Die Frankfurter Allgemeine Zeitung (FAZ) berichtet am gleichen Tag von „scharfe[r] Kritik an der Behörde für Cybersicherheit“ (2019, S. 1), nachdem durch einen schnöden Diebstahl die Daten von Hunderten Politikern, Netz-Aktivisten, Prominenten und Journalisten an die Öffentlichkeit gelangt waren. Bundestagsvizepräsident Kubicki (FDP), wie dessen Kollege Oppermann (SPD) schossen sich auf den Behördenchef ein: Man müsse sich fragen, ob er der richtige Mann für diese Position sei. Die Begründung der Vorwurfshaltung lag in einer, wie die Partei der Grünen es formulierte, ,,stark irritierenden Informationspolitik“ (Röpcke et al. 2019). Der Bundestagsvizepräsident Kubicki nahm vor allem an einer einzelnen Äußerung des Behördenchefs Anstoß. Er sagte: „Ein Präsident, der erst erklärt, man wisse seit Anfang Dezember von den Vorgängen, um jetzt zurückzurudern und zu sagen, man wisse es eigentlich erst seit dem 3. Januar" (FAZ 2019, S. 1), der müsse sich eben grundsätzlich infrage stellen lassen.

Lapsus linguae, eine Ungeschicklichkeit, ein Missverständnis... hier lag wohl der Empörungsgrund, genauer gesagt, der Anlass der Empörung. Jedermann konnte sich der Beiläufigkeit dieses Anlasses für den Vorwurf des Behördenversagens bewusst sein. Schließlich waren die fraglichen Daten gar nicht der Behörde

K. Kocks $(\square)$

CATO Sozietät für Kommunikationsberatung $\mathrm{GmbH}$, Horbach, Deutschland

E-Mail: ceterum.censeo@mailtrack.de 
gestohlen worden, sondern von einem jugendlichen Täter im Kinderzimmer der elterlichen Wohnung gesammelt, aus privaten Quellen mit mangelhaftem Schutz durch deren Eigner. Der Dienstherr stellte sich vor seine Behörde. Auf Spiegel Online war zu lesen:

\begin{abstract}
„Seehofer verteidigt Behörden nach Datenskandal (...). Nach dem Klau der Daten von Hunderten Prominenten und Politikern hat es den Vorwurf gegeben, die Behörden seien zu lange untätig gewesen. Innenminister Horst Seehofer nahm diese nun in Schutz. Die zuständigen Behörden hätten , sehr rasch, sehr effizient und sehr gut rund um die Uhr gehandelt", betonte der CSU-Politiker in Berlin bei einem gemeinsamen Auftritt mit dem Präsidenten des Bundesamts für Sicherheit in der Informationstechnik (BSI), Arne Schönbohm, und dem Präsidenten des Bundeskriminalamts (BKA), Holger Münch. Der 20 Jahre alte Tatverdächtige sei schon am vergangenen Sonntag identifiziert worden, sagte Seehofer und betonte: ,Wir machen in so wichtigen Angelegenheiten also unsere Arbeit. ' Dann erklärte der Innenminister, die Löschung der von dem mutmaßlichen Täter ins Internet gestellten Daten dauere an." (Hagen 2019)
\end{abstract}

Die „scharfe Kritik an Behörden“ (Rhein-Zeitung 2019) endete also wie das Hornberger Schießen, sicher war das nicht. Zunächst hatten Medien und endlose Kommentatoren in den Sozialen Medien des Internets die Behörden insgesamt diskreditiert und Köpfe rollen sehen wollen. Das Beispiel steht für die Gefahren einer ungeschickten Kommunikation einer öffentlichen Verwaltung, die sie zum Objekt von Reputationsverlust bis hin zu Rachegelüsten empörter Bürger, aber auch politischer Kontroversen machen kann, auch dann, wenn von einer wirklichen Verantwortung für die vermeintliche Misere nicht die Rede sein kann.

Öffentliche Verwaltung steckt immer zwischen Baum und Borke. Sie ist als Institution dem Staat wie der Politik verpflichtet, wendet sich aber andererseits an die Bürger, die nicht in allen Fällen jedem Begehren der Obrigkeit nachkommen wollen (Kloepfer 2012). Umgekehrt gilt diese Doppelrolle ebenfalls: Auch wenn Verwaltung sich als Bürgerservice versteht, so kann sie nicht in allen Fällen jedem Ansinnen jedes Betroffenen nachkommen, sie bleibt an Recht und Gesetz ebenso gebunden wie an politische Vorgaben (u. a.: Feik 2007; Gusy 2000; Schatz 2008). Dass sie also das ,Weltenkind in der Mitte“ ist, daran hat sich Verwaltung schon immer gewöhnen müssen. In autoritären Gesellschaften war sehr schnell mit dem ,Räsonnieren' Schluss und es wurde getan, was die Behörde wollte, weil der Staat eben dies vorgab. In demokratischen Gesellschaften lassen sich Meinungsbildungen in den Parlamenten und schon gar nicht in der Öffentlichkeit so ohne weiteres beenden (Sarcinelli 2011). Die Internet-Kommunikation, zu der sprichwörtlich jeder Zugang hat, hat hier viel 
verändert. Kritik kann auch dann sehr laut werden, wenn sie sachlich nur auf schwachen Füßen steht. Wenn es eine freie Presse gibt und sogenannte Soziale Medien im Internet, in denen ,Wutbürger' ihrem Ärger einen oft wilden Ausdruck geben, so muss Verwaltung oft für Dinge herhalten, die über das Bild von Baum und Borke hinausgehen. Denn zwischen Bürger und Verwaltung treten allemal die Medien, die auch Dritte, eigentlich Unbeteiligte, zu Meinungsträgern machen können (Krotz 2001). Das ,Weltenkind in der Mitte' kann den Einfluss der Medien auf beiden Seiten seiner Abhängigkeit bemerken, als ,Shitstorm' von Bürgern, aber auch als einen Teil der politischen Meinungsbildung, die überraschenden Änderungen unterworfen sein kann. Kurzum: Verwaltung ohne Kommunikation mit der Öffentlichkeit ist nicht (mehr) denkbar (Coleman und Blumler 2009; Schatz 2008; Stockinger 1983; Sweeney 2007).

Diese Interferenz von verwaltungsfachlichem Handeln und öffentlicher Wahrnehmung entspricht, um einen anderen Bereich zum Vergleich heranzuziehen, der von Verwaltung und Rechtsprechung. In vielen Dingen haben die Juristen das Sagen. Schon in den Verwaltungen ist ihre Stellung stark; geht es in streitigen Fällen vor Gericht, gilt endgültig ,iustitia non calculat', mit der Justiz ist schlecht zu handeln. Daher gibt es in der Folge eine ausgeprägte Rechtsberatung, ohne die keine Verwaltung mehr zurechtkommt. Dass es der Rechtsberatung für Verwaltungshandeln bedarf, das ist unstrittig. Was aber ist mit Kommunikationsberatung? Weit öfter als vor Gericht scheitert Verwaltung in der Öffentlichkeit durch eine böswillige Presse oder eine übel gelaunte Öffentlichkeit etwa. Was kann und was soll Politik- und Kommunikationsberatung leisten? Sie will den Handelnden der Verwaltung die Gesetzlichkeiten einer anderen Welt als der eigenen, eben jener der öffentlichen Meinungsbildung, nahebringen und so ein Scheitern in fremden Gefilden verhindern. Sie hat sich das Sprichwort zu eigen gemacht, dass schlimmer, als Unrecht zu haben, es sein kann, schlicht nicht verstanden zu werden. Diese Tragik ist allen Fachleuten vertraut, besonders virulent ist sie dort, wo Handeln zwischen Baum und Borke steckt.

Wenn man zu einem abgeklärten Bild dessen kommen will, was Politikund Kommunikationsberatung bewirken soll und auch erreichen kann, so wird man dieses ,consulting ' zunächst von dem Mythos befreien müssen, der ihm angedichtet worden ist. Der Mythos handelt von Politikberatern in der (Spitzen-) Politik, vornehmlich in Wahlkämpfen auf oberster Ebene. Deren ,fama' ist nicht alltagstauglich, möglicherweise nicht einmal zutreffend, kann aber eine realistische Wahrnehmung beeinträchtigen. Die legendären ,spin doctors' (in schönstem Deutschenglisch auch , spin meister ${ }^{6}$ ) sollen es verstanden haben, Wahlen zu entscheiden, indem sie ihre Klienten so berühmt machten, dass der Wähler gar nicht anders konnte, als ihnen seine Stimme zu geben (dazu u. a.: Bennett und Manheim 
2005; Marx 2008; McNair 2004; Savage und Tiffen 2007; Tenscher 2003). Der Autor dieser Zeilen hat viele der Legenden der Politikberatung persönlich kennenlernen dürfen; und er rät zu einer deutlich zurückhaltenderen Beurteilung.

Seit Mitte der 1960er Jahre hat in Deutschland Josef von Ferenczy, zumeist am eigenen Telefon (damals eine Sensation) aus der Bar des Hotels Vier Jahreszeiten in München, als Strippenzieher gewirkt. Er soll beispielsweise dem späteren Außenminister Hans-Dietrich Genscher zu dem berühmten gelben Pullunder geraten haben. Man erinnert sich, ein weiterer Name, an Bodo Hombach, der Johannes Rau in Nordrhein-Westfalen zu dem Wahlkampfslogan „Wir in NRW“ geraten hat. Beide Herren sind wirklich schätzenswert (Ferenczy ist inzwischen leider verstorben), aber Pullunder wie Slogan waren zwar von guter, aber durchaus beschreibbarer Qualität. Berühmter als diese deutschen Namen waren Dick Morris für den amerikanischen Präsidenten Bill Clinton und Alastair Campbell für den englischen Premierminister Tony Blair. Morris ist mittlerweile zu einem populistischen Aufwiegler derangiert, Campbell kämpft aus dem Off gegen die Pro-Brexit-Linie von Teilen seiner Partei. Beide waren zu ihren Zeiten und den Wahlkämpfen ihrer Kandidaten die internationale Vorhut des damals sensationellen ,spin doctoring'. In Deutschland wurden die Epigonen gehandelt, viele aus dem Journalismus kommend. Der ehemalige Chefredakteur Michael Spreng hat Edmund Stoiber unterstützt wie der BILD-Chef Hans-Hermann Tiedje Helmut Kohl. Beide Engagements waren kurz und eigentlich ist in der Sache wenig Sensationelles erinnerlich. Tempi passati. Man mag mit all diesen Namen eine gewisse Mystik der Macht verbinden, fachliche Gründe gibt es dafür nur sehr eingeschränkt. Man glaube dem Autor dieser Zeilen, der mit all den Genannten zu tun hatte, sie kochten mit Wasser. Die Annahme, dass daraus für eine alltagstaugliche Verwaltungskommunikation viel zu lernen sei, ist fehlleitend. Der Nimbus der ,spin doctors' ist ein Teil jener Propaganda, mit der man den politischen Erfolg glorifizieren will, der zu einem guten Teil schlicht den Zeiten oder dem Wählerwillen geschuldet war. Politik- und Kommunikationsberatung ist zu allererst ein Handwerk, nämlich jenes, welches der große Soziologe Niklas Luhmann beschrieben hat als „Reduktion von Komplexität“ (Luhmann 2009, S. 22). Das scheint wie eine sprachliche Aufgabe, es ist aber zuvor und im Kern eine intellektuelle.

Das Axiom der Politik- und Kommunikationsberatung bei Verwaltungshandeln klingt paradox, ist aber von wesentlicher Virulenz: Obwohl im allgemeinen Sinne geschehend, so ist Verwaltungshandeln nicht immer und nicht für jeden allgemein verständlich. Verwaltung ist weder für ihre, Vorgesetzten ' in der Politik noch für ihre ,Adressaten ' in der Zivilgesellschaft selbsterklärend. Das wäre weniger virulent, wenn ihr selbst dies bewusst wäre; leider entsteht ein derartiges Problembewusstsein meist erst in Fällen von eklatanten Missverständnissen. Dabei wird 
vergessen, dass die strukturell fehlende Plausibilität nachhaltig als ein schleichendes Gift wirkt. Im Bereich des Rechts entspricht dieser Satz der restringierten Plausibilität vielfältiger Erfahrung: Was geschriebenes Recht ist, muss nicht immer der Erwartung des Alltagsverstandes jedes Bürgers entsprechen. Von daher muss sich die Notwendigkeit von Rechtsberatung auch in der Regel nicht legitimieren. Bei Kommunikationsberatung ist das Moment der Anerkennung dieser Notwendigkeit geringer. Man unterstellt unter Fachleuten gern, dass die eigenen Einsichten auch von allen Laien geteilt werden, insbesondere die Fachleute unterstellen dies sich selbst. In autoritären Gesellschaften mag das hinnehmbar sein, weil man ,Sinn“ durch ,Macht` ersetzen kann. Demokratische Gemeinwesen tun sich da schwerer. Je stärker der Mitwirkungswille von Bürgern ausgeprägt ist, desto höher werden die Anforderungen an die spontane Plausibilität von Verwaltungshandeln.

Was muss Politik- und Kommunikationsberatung leisten? Sie muss Verwaltung verstehbar und verständlich machen können. Das ist in erster Linie eine Frage klarer Sprache, aber eben auch nachvollziehbarer Sachverhalte. Man könnte dies die kognitive, die verstandesmäßige Dimension von öffentlicher Kommunikation nennen. Fachsprache, wenn nicht gar Fachjargon, muss in verständliches Deutsch übersetzt werden. Dem geht aber eine intellektuelle Dimension voraus: Die Komplexität eines Sachverhaltes muss so reduziert werden, dass er noch zutreffend bleibt, aber gleichzeitig hinreichend verständlich wird. Dies ist nicht nur eine Frage der sogenannten ,einfachen Sprache', sondern der Nachvollziehbarkeit. Plausibilität herzustellen, verlangt zusätzlich zur kognitiven Dimension eine affektive. Das Thema muss eine kulturelle Kohärenz zur mentalen Disposition des Adressaten aufweisen. Die volkspädagogische Vorstellung, dass man für die ,Dummen' nur langsamer sprechen müsse (oder lauter, also schreien), ist eine Posse. Es geht um intersoziale Kompetenz. Werden andere Kulturkreise berührt, ist diese Herausforderung naheliegend, sie gilt im Grunde aber auch bei sozialer Distanz zwischen Kommunikator und Adressaten. Berater denken Kommunikationsaufgaben wie Übersetzungen in andere Sprachen, also in andere Kulturkreise; das ist eine Aufgabe der kommunikativen Inklusion. Fachsprachen, etwa das Lateinische der Medizin, dienen bewusst dem Gegenteil dieser Nachvollziehbarkeit; sie exkludieren Laien.

Der Fachjargon, der sich oft unbewusst einschleicht, hat aber den gleichen Effekt, nämlich Verstehbarkeit zu untergraben. Zu der kognitiven und affektiven Reduktion von Komplexität kommt ein drittes Kriterium, das erfolgreiche Kommunikation auszeichnet. Die vorgetragenen Sachverhalte müssen leicht erinnerbar sein. Memorabilität ist möglicherweise das wichtigste Kriterium gelingender Verständigung. Dass etwas einprägsam ist, wenn nicht gar ein ,Ohrwurm', entscheidet 
vor allem mittel- bis langfristig über den Grad der Verständigung. Reime, Slogans, Motti, Sprichwörter bilden hier das Paradigma. Ein historisches Beispiel mag das in Deutsch gesprochene Berliner Motto von John F. Kennedy (1963) bilden: „Ich bin ein Berliner“ war allgemein verständlich, prägnant, assoziationsstark und erinnerungsaktiv.

Alle bisherigen Überlegungen zu gelingender Kommunikation setzen voraus, dass die Kommunizierenden sich wohlmeinend gegenüberstehen und ein gemeinsames Interesse daran haben, zu einer Verständigung zu kommen. Dies ist die ,normale" Voraussetzung bei privaten Dialogen, die verständigungsorientiert sind. Für den politischen Raum ist dies aber eine naive, wenn nicht gar schlicht falsche Annahme, die zu verheerenden Folgen führen kann. Politischer Raum ist ein Feld des Machtkampfes, oft mit Gegnerschaft, manchmal mit regelrechten Feindesbeziehungen. In der Politik findet Kommunikation eben auch als Medium der Kontroverse statt, dabei gibt es ebenfalls den Willen, den politischen Gegner bewusst, nämlich vorsätzlich, falsch zu interpretieren, um daraus einen polemischen Angriff gestalten zu können. Deshalb gilt für eine derart politisch überformte Kommunikation: Man ist als Kommunikator auch für das Maß seiner Missverständlichkeit verantwortlich. Alte Hasen der parteipolitischen Kontroverse sprechen gar vom, Verhetzungspotenzial' einer Aussage. Man muss für die politische Kontroverse also eine prinzipielle Böswilligkeit unterstellen, weil man sie nicht bei allen Stakeholdern ausschließen kann. Empörungsprozesse, insbesondere in den Sozialen Netzwerken des Internets, entwickeln eine hohe Selbstständigkeit und Eigendynamik, in der der ursprüngliche Anlass der Empörung schnell vergessen sein kann und ein ,Shitstorm" die Fehlinterpretation zur Gewissheit werden lässt (dazu auch: Bergmann und Pörksen 2009).

Dabei kann es zu spiralförmigen Prozessen des Aufschaukelns in Intensität und Reichweite kommen. Da der Tenor hierbei zum Zorn und Hass gehen kann, nennen wir diese Dimension ,thymotisch', sie kann eine empathische Erwartung sehr schnell und leider auch sehr nachhaltig in eine hasserfüllte Antwortsituation verkehren. Man kennt Institutionen wie Positionen, die ihre Reputation nach einem solchen thymotischen Exzess als , verbrannt ${ }^{*}$ betrachten mussten. Von besonderer Bedeutung für diese kontraproduktive Wende sind Vorurteile bei den Adressaten, die unbeabsichtigt aktiviert werden. Oft unterschätzen Fachleute in den Verwaltungen die vorsätzliche Empfindlichkeit von ressentiment-affinen Milieus. In einer ressentiment-aversen Disposition gilt es, solche Rückschläge zu antizipieren. Das heißt, für eine gelingende Kommunikation diese schon im Ansatz zu vermeiden. Man wird die eigenen Aussagen daher auch mit den Augen des Teufels lesen müssen. 
$\mathrm{Zu}$ den Aspekten der Plausibilität und der semantischen Stabilität kommt die Dimension der mythischen Referenz. Oft erzählt man unbedacht mit einer sachlich gemeinten Geschichte andere große ,Stories' mit, die im kulturellen Resonanzboden einer Gesellschaft mitschwingen.

Was im Negativen gilt, gilt auch im Positiven. Große Bedeutung haben alle Kommunikationen erlangt, die historische Wurzeln haben und eine kulturelle Tiefenwirkung aufweisen. Der schon erwähnte Kennedy-Satz (1963) „Ich bin ein Berliner" zitiert den antiken römischen Leitspruch ,civis romanus sum" und damit das extraterritoriale römische Bürgerrecht. In Berlin als Leitspruch einer ,freien Welt' genannt, war dies eine klare Kampfansage an die Sowjetunion, deren Ostberliner Regime zwei Jahre zuvor den Mauerbau vorgenommen hatte. Der historische Tiefgang muss nicht allen Zuhörern auf dem Rathausplatz bei Kennedys Rede klar gewesen sein, begründet aber die Gewichtigkeit der Kommunikation mit: „All free men, wherever they may live, are citizens of Berlin“ (Kennedy 1963). Häufig finden wir Sprichwörter und Bibelzitate oder Anspielungen auf andere kulturelle Traditionen als Mittel gelingender Kommunikation. Jedenfalls gehört Konkulturalität zu dem, was hier zu beschreiben ist. Der Mythos um John F. Kennedy war so tief, dass das Weiße Haus zu seiner Zeit ,Camelot' genannt wurde. Das ist in den alten Mythen der Sitz von König Artus und den Rittern der Tafelrunde, die dort den Heiligen Gral hüten. Mehr Tiefe geht nicht.

Ein wesentlicher Teil der Politikberatung von Verwaltung muss in dem liegen, was die Strukturen und Prozesse der Politik ausmachen. Darüber soll hier, trotz der Bedeutung dessen, nicht berichtet werden. Wenn Beratung eine Übersetzungs- und Übertragungsleistung ist, so steht als noch eigengesetzlicherer Bereich der Transformation der der Medien an. Aus Gründen der größeren ,Farbigkeit' des Exempels soll hierauf exemplarisch eingegangen werden. Was also ist die Eigengesetzlichkeit des Medienprozesses, die Verwaltung entgegenschlägt? Die demokratische Institution einer, freien Presse` hat in jüngster Zeit ja einiges an Schädigung ihres eigenen Rufes erfahren müssen, Stichwort ,Lügenpresse'. Der Begriff ,Lügenpresse ' beruht auf einem ganz grundsätzlichen Irrtum, nämlich dem, dass es die ,Wahrheitspresse' geben könnte. Die Vorstellung der einen, nämlich der verbindlichen, Wahrheit, russisch prawda, geht aber nur, wenn man jemanden hat, der endgültig bestimmen kann, was wahr und was unwahr ist oder was eine richtige Meinung ist und was eine unrichtige sein soll. Diese Zeiten sind aber vorbei, jedenfalls sollten sie es sein. Ein Journalist darf von Berufs wegen alles, aber nicht langweilen. Das Brot seines Berufes sind Skandale.

Der frühere Bundeskanzler Willy Brandt, selbst gelernter Journalist, hat gesagt: „Der Journalismus kann abdanken, wenn er harmlos wird.“ Der Journalismus war früher, auch ein Wort von Willy Brandt, ein Teil der holzverarbeitenden 
Industrie, denn er verkaufte Papier. Nun kann man sich leicht vorstellen, dass an einem Tag, an dem die Welt in Ordnung ist, die Sonne scheint und es nichts gibt, worüber man sich empören könnte, der Journalist in einer wirklichen Not ist. Er kann nämlich kein bedrucktes Papier verkaufen, wenn dort nichts Sensationelles und Skandalöses vermeldet wird. Eine Boulevardzeitung, die am Kiosk liegt, auf der man nur eine weiße Fläche findet, vielleicht mit dem kleinen Randvermerk „Leider ist heute nichts passiert“, eine solche Boulevardzeitung wird sich schlecht verkaufen. So freuen sich dann vielleicht Leser, weil sie so viel Raum für Notizen haben und nicht erst nach einem Notizblock suchen müssen, um ihre Einkaufsliste zu machen, aber ein Geschäft ist das nicht. Daher kommt das Sprichwort, nach dem man in der Zeitung von gestern nur noch den Fisch einwickeln kann.

Was für die Zeitungen und Zeitschriften gilt, gilt für die elektronischen Medien gleichermaßen, vor allem aber für das Internet, wo die sogenannten Sozialen Medien, Facebook, Twitter, Instagram, ihr Unwesen treiben. Auch wenn inzwischen einzelne Verwaltungen hier kommunizieren, muss man zu einer gewissen Vorsicht raten. Hier herrscht ein Jahrmarkt der Eitelkeiten, der aus jeder Banalität eine kleine Sensation machen möchte. Dies ist nicht das Amtsblatt. Erwachsene Menschen fotografieren ihr Essen oder ihre Katze oder sich selbst, vorzugsweise leicht bekleidet. Eine Welt auf der Jagd nach Skandalen - auch eine Welt im Banalen. Das ist nicht mehr das, was man früher unter einer anständigen Presse verstanden hat. Die Eigendefinition von Journalismus lautet: Pressewürdig (,fit to print') ist alles, was irgendjemand auf gar keinen Fall lesen möchte. Alles andere sei PR, Public Relations, also Agitprop, Agitation, Propaganda oder Werbung. Es gehört somit zum Wesen einer freien Presse, dass sie sich etwas herausnehmen möchte. Sie will den Herrschenden und deren Agenten, sprich der öffentlichen Verwaltung, auf die Finger schauen und auf die Füße treten. Pressefreiheit schließt für Journalisten die Freiheit ein, eben diese auch missbrauchen zu wollen, jedenfalls Ärger in Kauf zu nehmen. Und zu den Herrschenden, dazu gehört eben vor Ort nicht nur Washington, Moskau und Berlin, sondern auch jedwede Verwaltung. Eine freie Presse ist, das darf nicht überraschen, auf Krawall gebürstet. Sie sieht sich als eine Vierte Gewalt im Staat, vor der die anderen Respekt haben sollen. Dem Journalisten als Menschen ist daher eine gewisse strukturelle Arroganz nicht fremd. Er gehört im Zweifel zu den Guten, die den Bösen das Leben schwer machen sollen. So lautet das hehre Selbstverständnis. Im Alltag kann die Vierte Gewalt aber eben auch ein Schandmaul oder eine Dreckschleuder auf der Suche nach Skandalen sein.

Presse ist dabei ein Geschäft, also Ökonomie. Das Wesen von Wirtschaften im Sinne von Ökonomie ist die Verwaltung und Verwertung knapper Güter. Was aber ist das knappe Gut des Journalismus? Man könnte meinen, dass es Nachrichten 
sind oder Sachverhalte oder das jüngste Verwaltungshandeln. In Wirklichkeit ist das knappe Gut des Journalismus aber nicht die Nachricht, sondern die Geschichte, insbesondere der Skandal. Die Währung, mit der im Geschäft der Presse, also der Ökonomie der Medien, Wert geschaffen wird, ist Empörung. Wir können die gesamte Medienkommunikation, genauer gesagt, die gesamte Massenmedienkommunikation, als Empörungskommunikation bezeichnen. Ob in China ein Sack Reis umfällt, interessiert, das leuchtet jedem ein, schlicht niemanden.

Presse entstand im 15. Jahrhundert durch die Erfindung des Mainzers Gutenberg, den Buchdruck mit beweglichen Lettern. Diese Erfindung, auch schwarze Kunst genannt, wäre aber längst nicht so bedeutend geworden, wenn es nicht wenig später einen wirklichen Wutbürger gegeben hätte, der die neue Technik mit Inhalten befeuerte. Der wütende Martin Luther lieferte nicht nur durch die Übersetzung der Bibel ins Deutsche, sondern vor allen Dingen durch seine Flugblätter gegen die Kirche und namentlich den Papst den Stoff, aus dem dann die Reformation geschaffen wurde, deren Motor Empörung war. Eine Empörungskommunikation verwandelt alltägliche Tatsachen in moralische Grundsatzfragen. Es beginnt mit einer Stolperfalle in Form einer losen Platte auf einem Gehweg und endet mit einem Wutbürger, der die Welt vernichten will, ob des von ihm erlittenen Unrechts. Pressegeschichten transformieren Alltägliches in einen Kampf von Gut und Böse, sie sind ethisch expansiv. Das macht sie so gefährlich.

Es geht nicht mehr um die Frage, ob ein Verwaltungshandeln angemessen ist, sondern es geht immer um die Frage, wer im moralischen Sinne gut und wer im moralischen Sinne böse ist und damit eigentlich zur Vernichtung ansteht. Empörungskommunikation ist in sich fundamentalistisch. Sie will Zorn erzeugen und Hass säen, jedenfalls will sie vorübergehend aufregen. Für solche , Geschichten' braucht man Skandale. Und wenn man keine Skandale hat, muss man welche finden. Wenn man keine Skandale veritablen Ausmaßes findet, steigt die Neigung, sie mit der Lupe zu suchen. Oder gar zu erfinden. Deshalb sucht Journalismus nach Sensationen, Skandalen, unabhängig davon, wie plausibel uns die erscheinen mögen. Die journalistische Regel heißt: „Hund beißt Mann? Das ist keine Geschichte. Mann beißt Hund! Das IST eine Geschichte.“ Deshalb kann ein Hinweis an die Presse, dass irgendein Vorfall die absolute Ausnahme sei und in keiner Weise repräsentativ, nicht beruhigen. So kann eine Verwaltung einem Journalisten niemals eine Geschichte ausreden. Im Gegenteil: Was selten ist, das macht ihn gerade scharf. Die unmöglichsten Geschichten schaffen es ins Blatt, gerade die.

Der Ausruf des interessierten Lesers ist: „Unglaublich, aber wahr!“ Weil dieses die Lesererwartung ist, haben die Medien die Neigung entwickelt, diese nicht nur halbwegs zu bedienen, sondern sie auch noch zu steigern. Und das verführt 
anschließend Männer, die unbedingt in die Presse wollen, dazu, Hunde zu beiBen. Die Skandalisierung von Vorgängen, also die Transformation von Tatsachen in Moral, geschieht in unterschiedlichen Ebenen in der Presselandschaft unterschiedlich stark. Die sogenannte Boulevardpresse langt hier stärker zu, als man es von den seriöseren Titeln kennt, aber für alle gilt, dass nur der Skandal eine wirkliche Geschichte ist. Man muss bei diesem inneren Zwang zur Empörungskommunikation natürlich die kritische Frage nach dem Nutzen und nach den Schäden stellen. Der Nutzen liegt sicherlich darin, dass das Medium Aufmerksamkeit erringt. Der Lehrsatz heißt: „Der Kampf der Empörungskommunikation ist ein Kampf um die Aufmerksamkeit der Nutzer." Denn unter allen Gütern, die uns zur Verfügung stehen, können wir eines nicht beliebig vermehren, nämlich die Zeit, die wir zur Mediennutzung bereitstellen. Hier findet im Internet ein regelrechter Kampf statt, der die Zeiten, zu denen man ,online' ist, auszuweiten gedenkt. Man wird das bei der Generation unserer Kinder oder Enkelkinder beobachten können: Es besteht eine regelrechte Abhängigkeitsbeziehung zu Onlinemedien. Viele der ganz jungen Menschen nutzen nicht nur ein einzelnes Gerät, etwa ihr iPhone, sondern meist schon parallel zwei oder drei Internetzugänge.

Aber ganz gleich, wie weit man die Sucht nach Internetkommunikation treibt, irgendwann ist selbst die Zahl der Geräte endlich und damit der Markt für die entsprechenden Medien nicht mehr ausweitbar. Drei oder vier Geräte gleichzeitig für eine Person und das $24 \mathrm{~h}$ am Tag, da ist dann Schluss. Die Folge ist, dass zwischen den Medien ein Verdrängungswettbewerb herrscht. Man erlebt, dass die Internetmedien in diesem Wettbewerb um unsere Aufmerksamkeit einen unaufholbaren Vorsprung erlangt haben. Wer die Nutznießer von Google und Co. sind, weiß man; man kennt die Gesichter der neuen Milliardäre, vornehmlich in den USA, aber inzwischen auch in China und anderen Ländern: big business. Hier ist der vordergründigste Nutzen in einem Kapitalismus, an dem Karl Marx seine Freude gehabt hätte. Der Schaden der Empörungskommunikation entsteht oft bei den dort behandelten Menschen und Themen. Dazu kann man einen wissenschaftlichen Blick auf den Prozess werfen, der hier abläuft. Wir haben es mit einem Zwang zur ,paradoxen Endoxie` zu tun (dazu auch: Kocks 2001).

Das Paradoxe ist der Aufhänger der Sensationsberichterstattung. Es passiert etwas, das man wirklich nicht erwartet hat, das unsere Aufmerksamkeit erregt, das uns bei Wahrnehmung empört erscheinen lässt und so das Weiterlesen oder Weiterzuschauen oder Weiterzuhören beflügelt. Der Skandal ist als solcher paradox, weil wir ihn nicht erwartet haben. Nun wird man einwenden, dass in den Boulevardmedien nicht jeden Tag etwas wirklich Neues steht, sondern wir irgendwann an die Mechanismen der Empörungskommunikation so gewöhnt sind, 
dass man schon eine feste Erwartungshaltung hat, die in vielen Fällen zu einer regelrechten Abnutzung der Aufmerksamkeit führt. Das hängt mit einem zweiten Bewegungsmoment zusammen, der in dem Skandal liegt. Auf das Paradoxe, das unsere Aufmerksamkeit erregt hat, folgt das Endoxe. Unter Endoxie versteht man Dinge, die unserem Glauben, unserem Vorurteil, unserer Erwartung entsprechen, unser Bauchgefühl, den gesunden Menschenverstand bestätigen. Das Paradoxe widerspricht unserer Erwartung, jedenfalls scheinbar. Das Endoxe erfüllt sie. Alle Boulevardgeschichten enden mit ein und demselben Schluss, dass wir uns als Leser in unseren Vorurteilen erneut bestätigt sehen. Man erfährt nicht wirklich etwas Neues, man wird nicht wirklich klüger, man weiß nicht wirklich nach Lektüre der Skandalgeschichte mehr über die Welt, das Leben oder die Nachbarn. Man hat sich empören können und findet sich am Ende des kurzen Vergnügens in seinen Vorurteilen bestätigt. Dies ist übrigens für Menschen ein ausgesprochen zufriedenstellender Vorgang. Wir lieben es, uns über andere aufzuregen, um am Ende recht zu behalten. Aus dem Wutschrei „Unmöglich!“ wird die tiefe Befriedigung, es schon immer gewusst zu haben. Wir sind von Vorurteilen geleitete Tiere. Die paradoxe Endoxie ist eine kreisförmige Bewegung. Sie funktioniert nach dem Mechanismus ,kaum zu glauben, aber wahr!“ Bei den Intensivnutzern der Medien kann eine regelrechte Sucht nach solchen Skandalisierungen entstehen.

Das aber ist hier nicht unser Thema. Die Frage ist, was den in der Verwaltung in Verantwortung stehenden Menschen diese Tendenz zur Empörungskommunikation antut. Aus dem bisher Gesagten ist schon klar, dass Vorgänge, für die es eine alltägliche Erklärung gäbe und damit einen Grund, sich zu beruhigen, trotzdem skandalisiert werden und so aus den ,ganz normalen' Sachbezügen in eine Welt der moralischen Wertunterschiede überführt werden. Aus Alltag wird Moral. Es gibt plötzlich gute Menschen, die Opfer einer Angelegenheit sind (die ,Bürger'), und böse Menschen, die die Täter einer Untat sind, also zur Rechenschaft gezogen werden müssen (die ,Bürokraten'). Das Dumme bei den hier behandelten Themen ist, dass die in einer Verwaltung arbeitenden Menschen selten in der Rolle der unschuldigen Opfer, also der Guten sind, sondern meist in die Rolle der Übeltäter gedrängt werden. Da schlagen die Dinge hohe Wellen, aber es sind eben nicht immer Wellen, die zur wirklichen Lösung des Problems einen Beitrag leisten können.

Folgt die Frage: Darf die Presse das? Wir müssen damit nach dem juristischen Rahmen der Presse fragen, aber auch nach den philosophischen Urgründen. Der philosophische Kern der Presse und damit der Pressefreiheit ist die sogenannte Meinungsfreiheit. Der Begriff wirkt allerdings schon stark einschränkend, weil es nicht nur um Meinungen geht. Im Englischen sprechen wir von Ausdrucksfreiheit, 
right of expression. Dies ist ein Grundrecht der Moderne, das davon ausgeht, dass jeder Mensch ein unverwirkbares Recht hat, seine eigene Persönlichkeit zu äußern, öffentlich darzustellen. Dies ist ein freies Land, jedenfalls sollte es ein freies Land sein, in dem es keine Einschränkungen des Individuums gibt, jedenfalls sollte es sie nicht geben. Das Recht gilt für jedermann, seine Persönlichkeit nach eigenem Gutdünken darzustellen. Dies kann durch eine Rede geschehen, dies kann durch künstlerische Tätigkeiten geschehen, dies kann durch die Art und Weise, wie man sein Leben führt, geschehen. Dieser philosophische Gedanke, der ein Menschenrecht formuliert, ist typisch für die Moderne. Das heißt, wir finden ihn in der schon genannten Reformation, also dem 16. Jahrhundert, aber staatstragend dann wirklich Ende des 18. Jahrhunderts. Das Recht auf einen freien Ausdruck seiner Persönlichkeit ist bahnbrechend im ersten Verfassungszusatz von 1791 zur amerikanischen Verfassung von 1776 formuliert. Und natürlich in unserer Verfassung, dem Grundgesetz von 1948. Es heißt dort: „Jeder hat das Recht, seine Meinung in Wort, Schrift und Bild frei zu äußern und zu verbreiten und sich aus allgemein zugänglichen Quellen ungehindert zu unterrichten. Die Pressefreiheit und die Freiheit der Berichterstattung durch Rundfunk und Film werden gewährleistet. Eine Zensur findet nicht statt" (Bundestag 2019).

Das Grundrecht der Pressefreiheit beinhaltet also ein Abwehrrecht des Bürgers gegen staatliche Eingriffe, aber eben auch eine institutionelle Bestandsgarantie für die Presse selbst. Der Schutzbereich des Grundrechtes der Pressefreiheit umfasst die gesamte Tätigkeit der Presse, von der Nachrichtenbeschaffung bis hin zur Veröffentlichung von Tatsachen und Meinungen. Geschützt ist dabei nicht nur der redaktionelle Teil eines Presseerzeugnisses, sondern auch der Anzeigenteil. Die Pressefreiheit bedeutet deshalb auch, dass Ausrichtung, Inhalt und Form des Presseerzeugnisses frei bestimmt werden können; zugleich, dass Informanten geschützt werden und das Redaktionsgeheimnis gewahrt bleibt. In der Weltrangliste der faktischen Pressefreiheit steht Deutschland auf Platz 15, Norwegen auf Platz 1. Die Pressefreiheit unterscheidet nicht zwischen seriöser Presse und Boulevardmedien. Das inhaltliche Niveau kann in der Abwägung mit anderen Rechtsgütern eine gewisse Rolle spielen, wo lediglich der oberflächlichen Unterhaltung dienende Presseerzeugnisse unter Umständen weniger ins Gewicht fallen als ernsthafte Darstellungen. Aber dies ist nur eine schwache Einschränkung; die Pressefreiheit gilt, um es umgangssprachlich zu sagen, für gute Presseerzeugnisse und für nicht so gute und auch sehr schlechte. Insbesondere hat der Staat kein Recht zu entscheiden, was ein gutes Presseerzeugnis ist, ein weniger gutes oder ein schlechtes. $\mathrm{Zu}$ gut deutsch: Wir müssen auch mit den Dreckschleudern leben. Ein Preis der Freiheit. Man kann sich gegen eine böse Berichterstattung wehren, aber leicht ist das nicht. 
Leitmotivisch sei eine Äußerung des englischen Satirikers Rowan Atkinson zitiert. Atkinson hat in englischer Tradition der Pressefreiheit folgendes gesagt: „Das Recht zu beleidigen steht über dem Recht, nicht beleidigt zu werden“ (Kocks 2019). Es gibt daher einen strukturellen Vorteil des verwalteten Bürgers gegenüber der Verwaltung. Die Pressefreiheit gilt zudem auch für Organe, die uns unter Umständen als Lügenpresse erscheinen. Auch die Lügenpresse hat das Recht auf ihrer Seite. Nicht für jede Lüge, was noch zu erörtern sein wird. Heißt das etwa, dass Presse lügen darf? Eine sehr, sehr gute Frage. Richtig ist, dass die Pressefreiheit nicht schrankenlos gewährleistet wird. Der Artikel 5 des Grundgesetzes nennt Schranken, nämlich die Vorschriften der allgemeinen Gesetze, die Bestimmungen zum Jugendschutz und das Recht der persönlichen Ehre; wie oben zitiert (Bundestag 2019). Umgangssprachlich gesagt, darf Presse nicht illegal werden. Es gibt aber eine Vielzahl von Gesetzesverletzungen, insbesondere dort, wo Journalisten ihre Informationen beschaffen wollen. Man muss zur Kenntnis nehmen, dass die Presse sich selbst als Vierte Gewalt sieht und daraus in vielen Fällen das Recht ableitet, fünf gerade sein zu lassen. Hier entsteht ein besonderer Beratungsbedarf von öffentlicher Verwaltung.

Auch wenn die Medienlandschaft als ,terra incognita' gelten mag, als fremder Kontinent, den zu betreten mit Wagnissen verbunden ist, so kann doch die erschienene Presse, das erfolgte Medienecho als eine gesicherte Basis für verlässliche Aussagen gelten. Mit den Methoden der Medienresonanzanalyse kann man wirklichen Boden unter den Füßen gewinnen. Nicht nur die aktuelle Rezeption wird klar, auch die Dispositionen der Teilnehmer und die aus den Strukturen folgenden Prozesse der Meinungsbildung. Eine kluge Kommunikationsberatung wird deshalb großen Wert auf die Evaluation ihrer Empfehlungen legen, um einen eher akzidentiellen Beratungsprozess in eine systematische Strategie überführen zu können.

Wenn Kommunikation einer ausgefeilten Strategie folgen sollte, so erwartet man mehr als eine Abfolge kluger Gedanken. Der strategische Anspruch weckt die Vorstellung einer Inszenierung. Ob eine öffentliche Verwaltung aber zur Durchsetzung ihrer Informationsbedürfnisse eine regelrechte Kampagne führen sollte, oder auch nur darf, das mag zweifelhaft sein, jedenfalls bedarf es einer Architektur der eigenen Informationsanstrengungen. $\mathrm{Zu}$ den Voraussetzungen für eine gelingende Kommunikation müssen eine Dramatik und Dramaturgie zustande kommen, die die eigentliche ,performance" sicherstellen. An dieser Stelle verlässt unsere Argumentation den Bereich einer Einführung in den Gegenstand ,Politik- und Kommunikationsberatung ' und darf mit Fug und Recht abgebrochen werden. Wir haben einen Ausblick genommen auf den unbekannten Kontinent der Medienlandschaft und könnten diese Übung wiederholen durch 
eine Expedition auf einen anderen Kontinent, den der Politik. Auch hier würden sich Eigengesetzlichkeiten zeigen, die nicht in der spontanen Erwartung einer Verwaltung liegen, also der externen Expertise bedürfen.

Fassen wir zusammen: Verwaltungshandeln trifft in der Öffentlichkeit auf Kausalitäten, die nicht in der eigenen DNA liegen. Dabei können Medienprozesse und politische Verzerrungen die ursprüngliche Intention bis zur Unkenntlichkeit entstellen oder schlicht in ihr Gegenteil verkehren. Aufgabe von Politik- und Kommunikationsberatung ist es, hier externe Expertise anzubieten, die den guten Willen zum gerechten Ziel führt.

\section{Literatur}

Bennett, W. L., \& Manheim, J. B. (2005). The big spin: Strategic communication and the transformation of pluralist democracy. In L. W. Bennett \& R. M. Entman (Hrsg.), Mediated politics. Communication in the future of democracy (5. Aufl., S. 279-298). Cambridge: University Press.

Bergmann, J., \& Pörksen, B. (2009). Skandal! Die Macht öffentlicher Empörung. Köln: Halem.

Bundestag. (2019). Die Grundrechte. https://www.bundestag.de/parlament/aufgaben/rechtsgrundlagen/grundgesetz/gg_01-245122. Zugegriffen: 30. Mai 2019.

Coleman, S., \& Blumler, J. G. (2009). The internet and democratic citizenship. Theory, practice and policy. Cambridge: University Press.

FAZ. (7. Januar 2019). Scharfe Kritik an der Behörde für Cybersicherheit. Frankfurter Allgemeine Zeitung.

Feik, R. (2007). Öffentliche Verwaltungskommunikation. Öffentlichkeitsarbeit, Aufklärung, Empfehlung, Warnung. Wien: Springer.

Gusy, C. (2000). Verwaltung durch Information. Empfehlungen und Warnungen als Mittel des Verwaltungshandelns. Neue Juristische Wochenschrift, 977-986.

Hagen, K. (8. Januar 2019). Seehofer verteidigt Behörden nach Datenskandal. Spiegel Online. https://www.spiegel.de/politik/deutschland/horst-seehofer-verteidigt-behoerden-nach-hackerskandal-a-1247017.html. Zugegriffen: 1. Juni 2019.

Kennedy, J. F. (26. Juni 1963). Remarks of the President John F. Kennedy at the Rudolph Wilde Platz, Berlin. https://www.jfklibrary.org/archives/other-resources/john-f-kennedy-speeches/berlin-w-germany-rudolph-wilde-platz-19630626. Zugegriffen: 1. Juni 2019.

Kloepfer, M. (2012). Staatsrecht kompakt. Staatsorganisationsrecht - Grundrechte Bezüge zum Völker- und Europarecht. Stuttgart: UTB.

Kocks, K. (2001). Glanz und Elend der PR. Das Verhältnis von Theorie und Praxis der Public Relations. Wiesbaden: Springer VS.

Kocks, K. (2019). Vierte Gewalt oder Lügenpresse? Von der Alltagsgegenwart der Propaganda. PR-Journal. https://pr-journal.de/fragen-und-meinungen/autoren-beitraege-themen-der-zeit/22254-vierte-gewalt-oder-luegenpresse-von-der-alltagsgegenwart-der-propaganda.html. Zugegriffen: 1. Juni 2019. 
Krotz, F. (2001). Die Mediatisierung kommunikativen Handelns. Wie sich Alltag und soziale Beziehungen, Kultur und Gesellschaft durch die Medien wandeln. Wiesbaden: Springer VS.

Luhmann, N. (2009). Zur Komplexität von Entscheidungssituationen. Soziale Systeme, $15(1), 3-35$.

Marx, S. (2008). Die Legende vom Spin Doctor. Regierungskommunikation unter Schröder und Blair. Wiesbaden: VS Verlag.

McNair, B. (2004). PR must die. Spin, anti-spin and political public relations in the UK, 1997-2004. Journalism Studies, 5(3), 325-338.

Prantl, H. (7. Januar 2019). Hackerangriff: Erwachet! Süddeutsche Zeitung.

Rhein-Zeitung. (7. Januar 2019). Regierung will Konsequenzen aus Online-Angriffen ziehen. Rhein-Zeitung. https://www.rhein-zeitung.de/deutschland-und-welt/newsticker_ artikel,-regierung-will-konsequenzen-aus-onlineangriffen-ziehen-_arid,1919166.html. Zugegriffen: 30. Mai 2019.

Röpcke, J., Solms-Laubach, F., \& Tiede, P. (7. Januar 2019). Daten-Angriff auf Prominente und Politiker. Die Jagd auf den Hacker. Bild.

Sarcinelli, U. (2011). Arenen parlamentarischer Kommunikation: Vom repräsentativen zum präsentativen Parlamentarismus? In U. Sarcinelli (Hrsg.), Politische Kommunikation in Deutschland. Medien und Politikvermittlung im demokratischen System (S. 263-282). Wiesbaden: VS Verlag.

Savage, S., \& Tiffen, R. (2007). Politicians, journalists, and ,spin“: Tangled relationships and shifting alliances. In S. Young (Hrsg.), Government communication in Australia (S. 79-92). Cambridge: University Press.

Schatz, H. (2008). Regieren in der Mediengesellschaft: Zur Medialisierung von Politik und Verwaltung in der Bundesrepublik Deutschland. In W. Jann \& K. König (Hrsg.), Regieren zu Beginn des 21. Jahrhunderts (S. 127-174). Tübingen: Mohr Siebeck.

Stockinger, H. G. (1983). Bürgernahe kommunale Öffentlichkeitsarbeit. Hilfen für Mandatsträger $u$. Verwaltung. München: Hanns-Seidel-Stiftung.

Sweeney, A. D. P. (2007). Electronic government-citizen relationships. Exploring citizen perspectives. Journal of Information Technology \& Politics, 4(2), 101-116. https://doi. org/10.1080/19331680802076165.

Tenscher, J. (2003). Mythos „Spin Doctors“: Analytische Anmerkungen und empirische Befunde zu Zentralakteuren moderner Politikvermittlung. In U. Sarcinelli \& J. Tenscher (Hrsg.), Machtdarstellung und Darstellungsmacht (S. 69-86). Baden-Baden: Nomos. 
Open Access Dieses Kapitel wird unter der Creative Commons Namensnennung 4.0 International Lizenz (http://creativecommons.org/licenses/by/4.0/deed.de) veröffentlicht, welche die Nutzung, Vervielfältigung, Bearbeitung, Verbreitung und Wiedergabe in jeglichem Medium und Format erlaubt, sofern Sie den/die ursprünglichen Autor(en) und die Quelle ordnungsgemäß nennen, einen Link zur Creative Commons Lizenz beifügen und angeben, ob Änderungen vorgenommen wurden.

Die in diesem Kapitel enthaltenen Bilder und sonstiges Drittmaterial unterliegen ebenfalls der genannten Creative Commons Lizenz, sofern sich aus der Abbildungslegende nichts anderes ergibt. Sofern das betreffende Material nicht unter der genannten Creative Commons Lizenz steht und die betreffende Handlung nicht nach gesetzlichen Vorschriften erlaubt ist, ist für die oben aufgeführten Weiterverwendungen des Materials die Einwilligung des jeweiligen Rechteinhabers einzuholen.

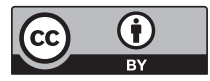

